\title{
Imaging of water droplet clusters on glass with simultaneous laser-induced fluorescence and near-infrared absorption
}

\author{
Florian Mirschinka*1, Jan N. Geiler ${ }^{1}$, Sebastian A. Kaiser², \\ Matthias Mansbart ${ }^{1}$, Erik Schünemann ${ }^{1}$ \\ ${ }^{1}$ Powertrain Solutions, Robert Bosch GmbH, Renningen 71272, Germany \\ ${ }^{2}$ Institute for Combustion and Gas Dynamics - Reactive Fluids, \\ University of Duisburg-Essen, Duisburg 47057, Germany \\ *Corresponding author email: Florian.Mirschinka@de.bosch.com
}

\begin{abstract}
Water films and droplets on surfaces are important in the design of various processes. In the present work, two complementary non-invasive imaging techniques, laser-induced fluorescence (LIF) and near-infrared absorption (NIRA), were used simultaneously to quantify the deposited water mass in a simple spray-onto-plate experiment. LIF was applied in epiillumination with optical access from only one direction, but it requires the addition of a marker substance to the non-fluorescing water. Here, anisole (methoxybenzene) was used for this purpose. The present implementation of NIRA is based on the intensity ratio of light transmitted through the sample at two wavelengths, 1450 and $600 \mathrm{~nm}$. Since water itself is the IR absorber, a tracer is not required. In a first step, in a thin-film cuvette the influence of temperature and film thickness on the measurement accuracy was investigated for both techniques. In a second set of experiments, water was sprayed onto a hot or cold quartz glass plate and simultaneously imaged with both techniques. From the measurements, advantages and weaknesses of both approaches were identified.
\end{abstract}

\section{Keywords}

Laser-induced fluorescence, near-infrared absorption, water wall wetting, anisole, tracer

\section{Introduction}

Liquid water droplet clusters and films are relevant in many industrial processes where either wall wetting is to be used or avoided. In gasoline engines, for example, water can be injected to prevent irregular combustion phenomena. To cool the fuel-air mixture most effectively, the water spray should not wet the walls. Optimization of such systems requires the characterization of water films. Among the techniques capable of two-dimensional imaging are laser-induced fluorescence (LIF) and near-infrared absorption (NIRA).

The main advantages of LIF are its high sensitivity, which allows measuring water thicknesses of less than $10 \mu \mathrm{m}$, and that optical access from only one side of the film is needed [1, 2]. However, LIF requires the addition of a marker substance, a so called "tracer", to the nonfluorescing water. Greszik [3] dissolved benzyl alcohol, ethyl acetoacetate, and urea as potential tracers in water and investigated the LIF signal upon excitation at $266 \mathrm{~nm}$. Schagen [4] dissolved diacetyl in water to measure mass and heat transport in laminar-wavy fluid layers using fluorescence and phosphorescence excited at $405 \mathrm{~nm}$. To image liquid water in an optically accessible engine Mirschinka et al. [5] used anisole as tracer that was excited at $266 \mathrm{~nm}$. Winter [6] investigated the air-sided mass boundary layer at the wind-driven airwater interface. He compared LIF signals of 1,4-difluorobenzene, acetone, anisole and other species on the air side at an excitation wavelength of $266 \mathrm{~nm}$. However, he observed that the tracers also dissolved in the water. The tracers most frequently used in literature for water LIF, such as Eosin $\mathrm{Y}$, rhodamine $\mathrm{B}$, or rhodamine $6 \mathrm{G}$, do not co-evaporate with water due to their 
high boiling points. Volkov and Strizhak [7] found that the proportion of rhodamine B dissolved in water increases during evaporation. Tracer-based measurements then do not accurately represent the solvent anymore.

Fuel-film imaging via LIF is well established. In recent studies by Schulz et al. [1, 8] and Geiler et al. [9, 10], iso-octane/tracer films were imaged in a pressure vessel and on the piston top surface of an optically accessible engine upon excitation at $266 \mathrm{~nm}$, respectively. In contrast to LIF, infrared absorption and Raman scattering are tracer-free methods. However, their signal-to-noise ratios are significantly lower [3]. Greszik et al. [11, 12] measured the thickness of contiguous, smooth water films with Raman scattering, infrared absorption, and LIF. The results showed approximately the same thicknesses measured by Raman as by LIF, the latter with ethyl acetoacetate as a tracer excited at $266 \mathrm{~nm}$.

Lubnow et al. [13] imaged water films sandwiched between a substrate and a transparent cover plate using NIRA. They used a fast-framing near-infrared (NIR) camera and a pair of time-multiplexed distributed feedback (DFB) diode lasers to switch between the absorption $(1469 \mathrm{~nm})$ and transmission (1353 nm) wavelength. Dupont et al. [14] imaged the thickness of falling water films on a glass plate by detecting back-reflected NIR light at $1612 \mathrm{~nm}$.

In previous studies, LIF was used either to study contiguous water films thicker than $500 \mu \mathrm{m}$ or to determine the temperature of water droplets (typically with rhodamines as tracers). Imaging IR was performed only for contiguous water films. Here, instead, evaporation of water droplet clusters with water thicknesses less than $300 \mu \mathrm{m}$ were imaged simultaneously using LIF and NIRA. This allows a direct comparison of advantages and disadvantages of the two methods, some of which are specific to the geometry of droplets as opposed to films.

\section{Methods \\ Experiment}

The experiment for simultaneous LIF and NIRA imaging is shown in Figure 1. Demineralized water with anisole as tracer was sprayed laterally via an injector onto a quartz glass plate at either room-temperature or heated to $343 \mathrm{~K}$. For some experiments, the quartz plate was replaced by a custom-built heatable thin-film cuvette with a clear aperture of about $30 \mathrm{~mm}$. This device allowed setting defined film thicknesses between 0 and $300 \mu \mathrm{m}$ by placing precision spacer shims between two quartz windows.

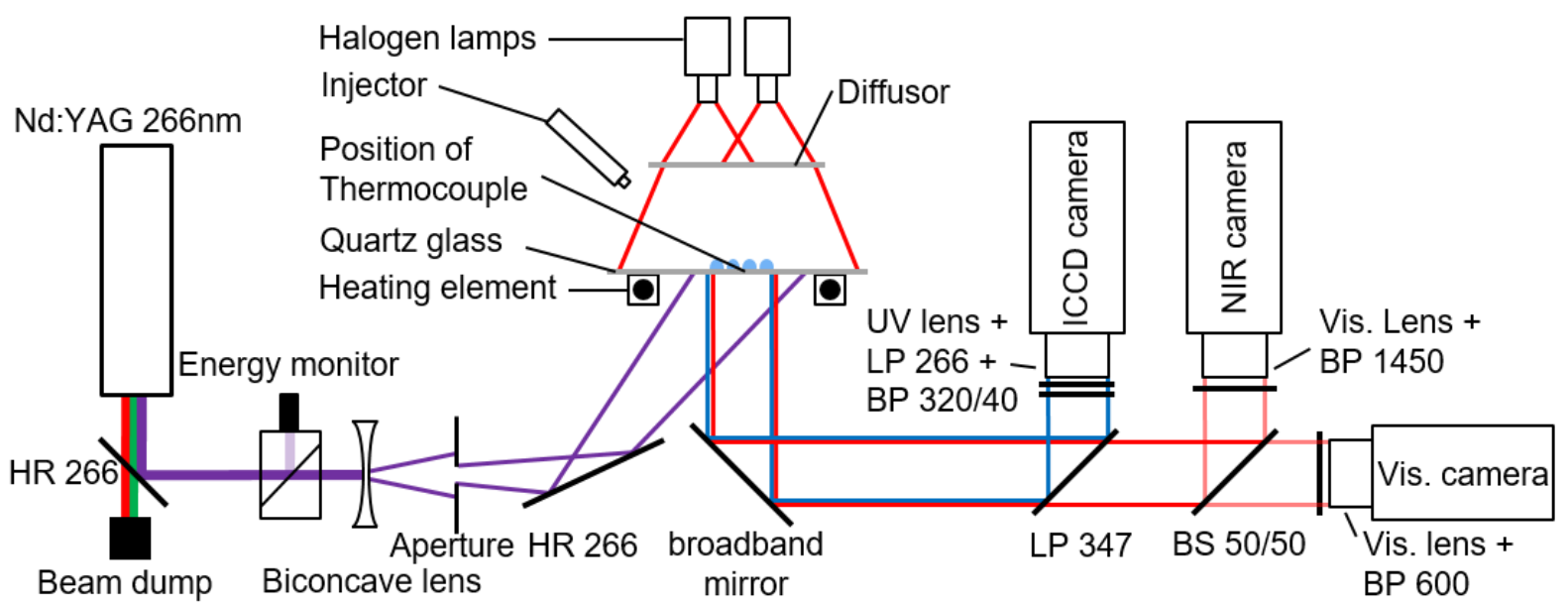

Figure 1. Optical layout for simultaneous visualization of water via LIF and NIRA.

For tracer LIF, a $266 \mathrm{~nm}$ mirror separates the fourth harmonic $(266 \mathrm{~nm})$ of an Nd:YAG laser from the remaining second harmonic $(532 \mathrm{~nm})$. An energy monitor logs the energy of each 
laser pulse to correct for pulse-to-pulse variations of the total laser energy. The laser beam is expanded to illuminate the quartz glass plate with a fluence of $0.6 \mathrm{~mJ} / \mathrm{cm}^{2}$. A broadband mirror deflects the emitted fluorescence light towards a dichroitic beam splitter that reflects wavelengths shorter than $347 \mathrm{~nm}$ (LP 347). An intensified CCD (0.2 $\mu$ s exposure time) with a UV objective (Cerco, $100 \mathrm{~mm}, \mathrm{f} / 2.8$ ) detects fluorescence, while a $266 \mathrm{~nm}$ long pass filter and a $532 \mathrm{~nm}$ notch filter suppress scattered laser light. The NIRA imaging consists of two broadband lamps that illuminate the quartz plate via a diffusor. Transmitted through the LP 347, a 50/50 beam splitter directs the light into two detection channels at $600 \mathrm{~nm}$ (Vis. Camera) and at $1450 \mathrm{~nm}$ (NIR camera), both with $8 \mathrm{~ms}$ exposure time. Choosing one NIRA wavelength in the visible range has the advantage that significantly more camera models are available. The projected pixel size was $0.17 \mathrm{~mm} /$ pixel and the recording rate was $5 \mathrm{~Hz}$. The field of view has a size of $40 \times 60 \mathrm{~mm}$.

\section{LIF imaging}

For weak excitation, the detected fluorescence signal $S_{\mathrm{f}}$ can be described by equation (1), where $\Omega \cdot \eta$ denotes the collection and detection efficiency of the imaging system, and $\phi$ the fluorescence quantum yield (FQY). $I_{0}$ is the incident intensity, $\epsilon^{*}$ the molar extinction coefficient, $c_{\mathrm{Tr}}$ the molar tracer concentration, and $d$ the thickness of the water/tracer mixture. The FQY and the extinction coefficient in the liquid [3, 4, 10] and the gas-phase [15, 16] depend on the temperature $T$. Additionally, the FQY depends on the tracer concentration. Thus, in general, these influences on the fluorescence signal must be known to derive the water thicknesses from the LIF signal.

$$
S_{\mathrm{f}}=\Omega \cdot \eta \cdot \phi\left(T, c_{\mathrm{Tr}}\right) \cdot I_{0} \cdot\left(1-e^{-\epsilon^{*}(T) \cdot c_{\mathrm{Tr}} \cdot d}\right)
$$

All images were background-corrected and divided by the corresponding pulse energy to correct for shot-to-shot laser fluctuations. To account for spatial inhomogeneities in excitation, detection, and collection efficiencies, each image was divided by the fluorescence of a socalled flat-field (FF). For acquiring this FF, a Schott-glass long-pass filter WG280 (thickness $2 \mathrm{~mm}$ ) was placed where the water wets the glass. A more detailed description of the data processing and the calibration device is given in [9].

\section{NIRA imaging}

In NIRA imaging, one channel detects the signal at a wavelength at which relatively strong absorption occurs, here $1450 \mathrm{~nm}$. A second channel detects light at a wavelength of $600 \mathrm{~nm}$, at which liquid water shows relatively weak absorption. Equation (2), based on the BeerLambert law, describes the relation between the transmitted light intensity $I_{\mathrm{t}}^{\lambda}$, the water thickness $\mathrm{d}$, the incident light intensity $I_{0}^{\lambda}$, and the absorption coefficient $k_{\lambda}$. In equation (2), $u$ describes elastic transmission losses like scattering or reflection on surfaces [13]. Thus, the term (1-u) represents the optical transmission efficiency. Assuming that this transmission efficiency is independent of the wavelength, it can be eliminated by taking the ratio of measurements at two wavelengths, yielding equation (3). Knowing the absorption coefficients at 1450 and $600 \mathrm{~nm}$, the water thickness $\mathrm{d}$ can be calculated.

To illustrate why this dual-wavelength measurement is needed, Figure 2 shows simultaneously recorded images of a water droplet placed on the quartz glass detected at both NIRA wavelengths. At $600 \mathrm{~nm}$, most of the light is transmitted in the center of the droplet. However, areas near the edge of the droplet are dark due to light refraction. In contrast, the image at $1450 \mathrm{~nm}$ shows strong absorption over the whole droplet. Assuming that the refraction is approximately the same for both wavelengths (the refractive index of water at 
$600 \mathrm{~nm}$ is $\mathrm{n}_{600} \approx 1.33$ and $\mathrm{n}_{1450} \approx 1.32$ at $1450 \mathrm{~nm}$ ), refraction effects are accounted for by equation (3).

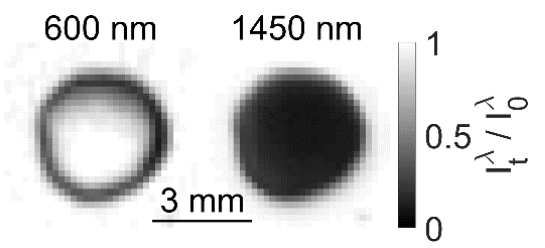

$$
\begin{aligned}
& I_{\mathrm{t}}^{\lambda}=(1-u) \cdot I_{0}^{\lambda} \cdot \exp \left(-k_{\lambda} \cdot d\right) \\
& d=\ln \left(\frac{I_{\mathrm{t}}^{1450} \cdot I_{0}^{600}}{I_{0}^{1450} \cdot I_{\mathrm{t}}^{600}}\right) \cdot \frac{1}{k_{1450}-k_{600}}[13]
\end{aligned}
$$

Figure 2. Simultaneously acquired transmission images of a droplet at 1450 and $600 \mathrm{~nm}$.

For NIRA imaging, the room light was switched off. A dark image was subtracted from all images. For the droplet measurements, the incident reference intensities $I_{0}^{1450}$ and $I_{0}^{600}$ in equation (4) were acquired by averaging images without water on the plate. $I_{\mathrm{t}}^{1450}$ and $I_{\mathrm{t}}^{600}$ represent images with wetted quartz glass. To be able to form the pixel-wise ratio of the NIRA images in equation (3), the images were geometrically precisely aligned ("registered").

\section{Results and discussion}

\section{Fluorescence properties of anisole in water}

In this work anisole (methoxybenzene, $\mathrm{C}_{7} \mathrm{H}_{8} \mathrm{O}$ ) was chosen as the fluorescent tracer for water. Anisole is liquid at room temperature with a solubility of $0.014 \mathrm{~g} / \mathrm{l}$ in water, and it has a boiling point of $427 \mathrm{~K}$. Figure 3 shows the spectral features relevant for LIF imaging. Transmission data to calculate the absorption cross section were measured with a PerkinElmer Lambda25 spectrometer in a $1 \mathrm{~mm}$ cuvette at a concentration of 0.0001 vol.\% anisole in water. The fluorescence spectrum was detected with a ICCD camera mounted at the exit plane of a grating spectrograph (Oriel MS260i, 50 lines $/ \mathrm{mm}$, blazed at $300 \mathrm{~nm}$, resolution $2 \mathrm{~nm}$ ). The thinfilm cuvette described above was used here.

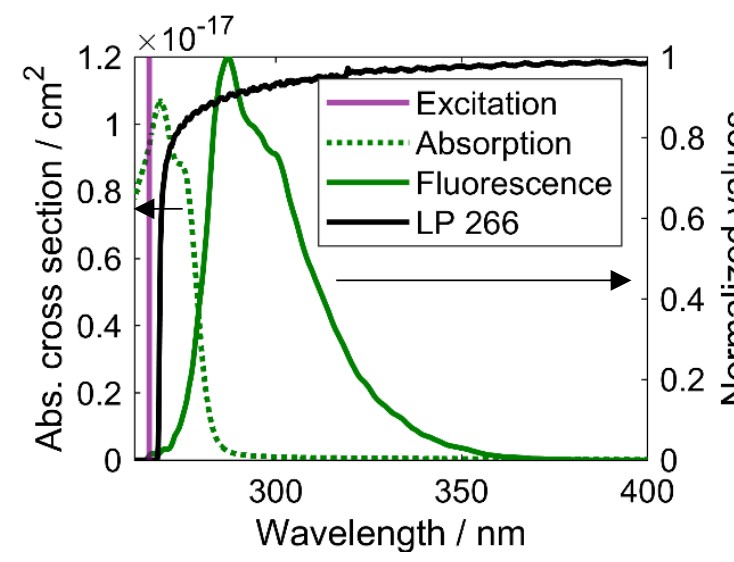

Figure 3. Peak-normalized fluorescence spectrum of anisole in water excited at $266 \mathrm{~nm}$, and absorption cross section as well as transmission of the long-pass filter [17].
Table 1 - Photo-physical properties of absorption and fluorescence of anisole dissolved in water at room temperature.

\begin{tabular}{|l|cc|}
\hline & This study & Literature \\
\hline$\sigma_{266 \mathrm{~nm}}$ & $9.4 \cdot 10^{-18} \mathrm{~cm}^{2}$ & $8.9 \cdot 10^{-18} \mathrm{~cm}^{2}[10]$ \\
$\lambda_{\sigma, \max }$ & $269 \mathrm{~nm}$ & $272 \mathrm{~nm}[10]$ \\
$\lambda_{\mathrm{S}_{\mathrm{f}, \max }}$ & $287 \mathrm{~nm}$ & $291 \mathrm{~nm}[18]$ \\
$\lambda_{\text {Stokes }}$ & $18 \mathrm{~nm}$ & $19 \mathrm{~nm}[18]$ \\
\hline
\end{tabular}

Table 2 gives an overview of the measured photo-physical properties and the corresponding values from literature. The measured absorption cross-section of anisole dissolved in water is $\sigma_{266 \mathrm{~nm}}=9.4 \cdot 10^{-18} \mathrm{~cm}^{2}$ at $266 \mathrm{~nm}$. The absorption maximum is at $\lambda_{\sigma, \max }=269 \mathrm{~nm}$. The fluorescence spectrum has a maximum at $\lambda_{\mathrm{lf}, \max }=287 \mathrm{~nm}$ and a Stokes shift of $\lambda_{\text {Stokes }}=18 \mathrm{~nm}$, 
the latter taken between absorption and fluorescence maximum. All of these values are in good agreement with the literature.

Figure 4 (a) shows the results of a variation in film thickness and tracer concentrations at room temperature and pressure in the thin-film cuvette. The intensity values in Figure 4 are the median of all pixel intensities within the field of view (FOV). Horizontal error bars indicate the sum of the thickness tolerances of the spacers and the roughness of the quartz glass windows. Vertical error bars indicate the standard deviation within the FOV. Solid lines represent a global fit to equation (1), in which the fitting parameter was $a=\Omega \cdot \eta \cdot \phi[9]$. The extinction coefficient calculated from the absorption cross section is $\varepsilon^{*}=5645\left(\mathrm{dm}^{3} / \mathrm{mol} \cdot \mathrm{cm}\right)$. Equation (1) describes the data well. For the imaging experiments described below, a tracer concentration of $\mathrm{c}_{\mathrm{Tr}}=0.006$ vol. $\%$ anisole dissolved in water was chosen.

Figure 4 (b) shows the result of a temperature variation. A $200 \mu \mathrm{m}$ thick film of water with 0.006 vol. \% anisole was heated from $293 \mathrm{~K}$ to $443 \mathrm{~K}$ and then cooled down back to room temperature. To avoid boiling, the cuvette was pressurized by nitrogen at $p=10$ bar. The data show that the signal decreases with increasing temperature. At $403 \mathrm{~K}$, the fluorescence signal is $49 \%$ of the room-temperature value. This is qualitatively consistent with literature results from gaseous anisole in nitrogen or carbon dioxide [19, 20]. For liquid anisole dissolved in isooctane Geiler [10] measured at $403 \mathrm{~K}$ a signal decrease to $50 \%$, and in iso-dodecane Mueller et al. [21] measured at the same temperature a decrease to $28 \%$.
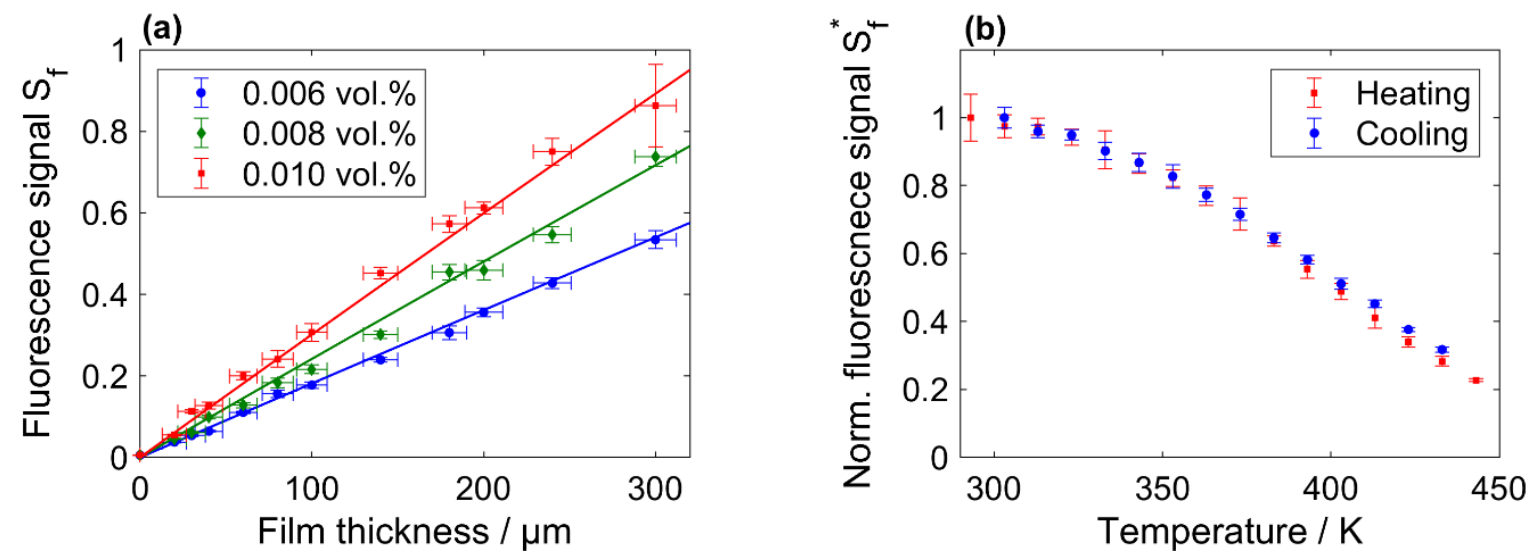

Figure 4. (a) Variation of film thickness and anisole concentration ( $p=1$ bar). Solid lines represent global fit and horizontal error bars indicate set thickness tolerance. (b) Variation of film temperature ( $\mathrm{c} T \mathrm{r}=0.006 \mathrm{vol} . \%$, $p=10$ bar, $d=200 \mu \mathrm{m})$. In both (a) and (b), vertical error bars indicate one standard deviation within the FOV.

\section{NIRA absorption coefficients and temperature dependence}

Figure 5 (a) shows the transmission in each detection channel and the resulting measured film thickness for different set thicknesses at room temperature and ambient pressure. Singlepixel intensity values and error bars were calculated as for LIF. Transmission fits are based on the Beer-Lambert law given in equation (2) with $\mathrm{k}_{\lambda}$ as fitting parameter and $u=0$. The straight black line with slope unity represents the set nominal film thickness. The diagram shows that water transmits almost all of the incident light at $600 \mathrm{~nm}$, whereas at $1450 \mathrm{~nm}$, water absorbs almost $60 \%$ at $300 \mu \mathrm{m}$ film thickness, and $90 \%$ absorption are reached at $800 \mu \mathrm{m}$ film thickness (not shown in the plot). Via equation (2), absorption coefficients of $\mathrm{k}_{600}=0.03 \mathrm{~cm}^{-1}$ at $600 \mathrm{~nm}$ and $\mathrm{k}_{1450}=28.6 \mathrm{~cm}^{-1}$ at $1450 \mathrm{~nm}$ were calculated from the transmission fits. With these absorption coefficients and equation (3), the calculated NIRA film thickness in Figure 5 (a) was determined. It shows good agreement with nominal thickness. 
The temperature dependency of transmission was investigated with a water film of $200 \mu \mathrm{m}$ thickness at ambient pressure. Figure $\mathbf{5}(\mathbf{b})$ shows that at all temperatures, within the measurement error transmission at $600 \mathrm{~nm}$ is equal to its room-temperature value, while at $1450 \mathrm{~nm}$ it slightly increases with temperature. At $343 \mathrm{~K}$, the transmission increases by $1.7 \%$ compared to its ambient-temperature value. Lubnow et al. [13] measured an increase of $6 \%$ at $338 \mathrm{~K}$ compared to room temperature value. A temperature-corrected absorption coefficient of $\mathrm{k}_{1450}=27.7 \mathrm{~cm}^{-1}$ was used to calculate the droplet thickness at $343 \mathrm{~K}$.
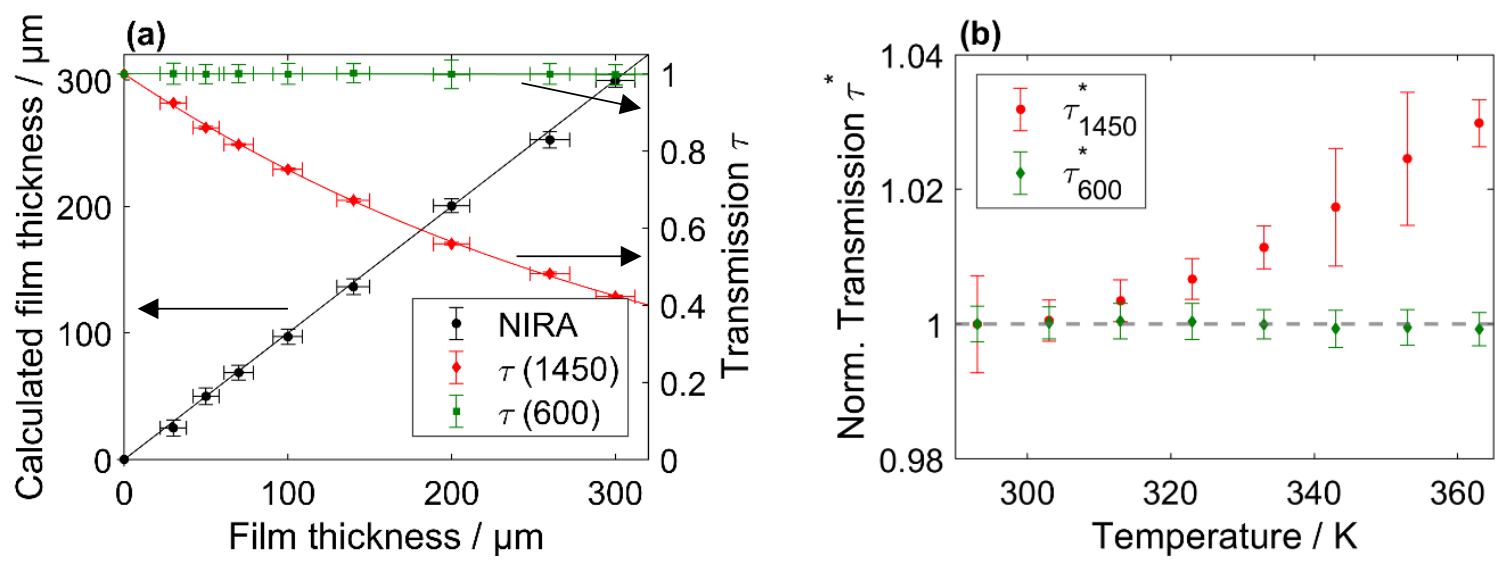

Figure 5. (a) Variation of film thickness ( $p=1$ bar). Horizontal error bars indicate set thickness tolerance. (b) Variation of temperature $(\mathrm{p}=1$ bar, $d=200 \mu \mathrm{m})$. Vertical error bars indicate standard deviation within FOV in both figures.

\section{Droplet clusters in simultaneous LIF and NIRA imaging}

Figure 6 shows a time series of an evaporating droplet cluster recorded simultaneously with LIF and NIRA imaging. Based on each technique's measurement noise, thicknesses less than $2 \mu \mathrm{m}$ (LIF) and $21 \mu \mathrm{m}$ (NIRA) were considered below the detection limit and were set to zero to improve visual clarity and increase accuracy in the field-wide mass calculation.

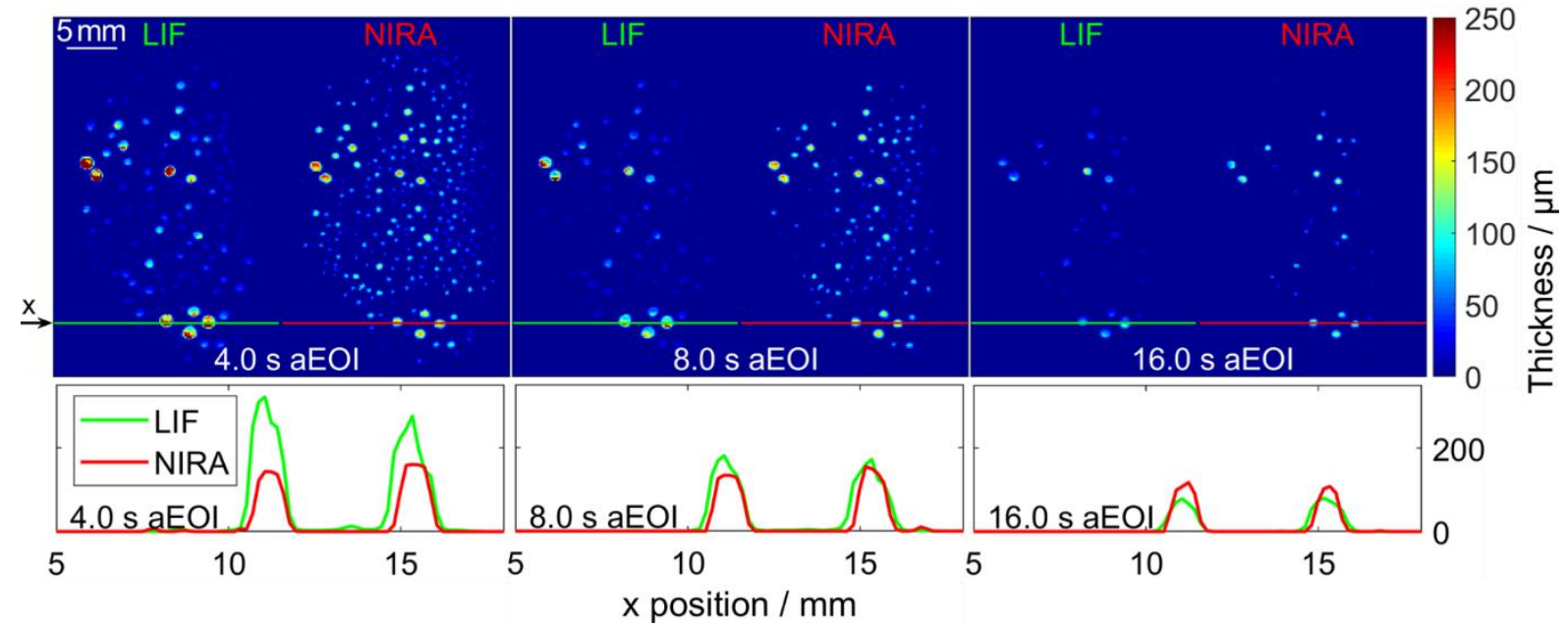

Figure 6. (Top row) Water droplet cluster from a spray impacting on a glass plate at $343 \mathrm{~K}$ with an injected water mass of $18 \mathrm{mg}$, imaged simultaneously with LIF and NIRA at three different times aEOI. (Bottom row) thickness profiles extracted along the lines marked by " $x$ " in the images.

Especially for big droplets, the images $4 \mathrm{~s}$ after end of injection (aEOI) show greater thicknesses for LIF than for NIRA, while $8 \mathrm{~s}$ aEOI thicknesses are approximately the same for both methods. $16 \mathrm{~s}$ aEOI most of the droplets have evaporated and the thicknesses of the remaining droplets from LIF are slightly smaller than NIRA thicknesses. The difference in LIF 
and NIRA for large droplets shortly aEOI can be attributed to two reasons, inaccuracies in the temperature determination for temperature correction, and light refraction within the droplets. Concerning the first issue, the LIF images were temperature-corrected by $16 \%$ whereas the NIRA images were corrected by only $3 \%$. Since the water temperature could not be measured, it was assumed to be that of the glass plate. However, since the water was at room temperature in the injector, it is actually initially cooler than the plate. Assuming too high a temperature, droplet thicknesses shortly aEOI are overestimated by both measurement methods but less so for NIRA. Figure 7 (a) shows averaged total water masses on heated and cold quartz glass. Green data points represent LIF and red ones NIRA. To calculate total masses, the thickness was integrated over all pixels and the results were multiplied with the projected pixel size and the water density at $343 \mathrm{~K}$ and $293 \mathrm{~K}$, respectively. The averaged values were calculated from 20 individual injections, and the error bars indicate the standard deviation over all single shots. As Figure 7 (a) indicates, the total evaporation time of the droplet cluster at $343 \mathrm{~K}$ was approximately $20 \mathrm{~s}$. At room temperature, the LIF-measured water mass decreases over time, in contrast to the NIRA data. Evaporation of anisole from the water can be an explanation for this. In addition, LIF yields significantly higher water masses than NIRA. Even (unphysical) values greater than the total injected mass are detected. At room temperature, this cannot be due to inaccuracies in the temperature correction. Instead, there may be a second reason for the overestimation by LIF, which will be discussed next.

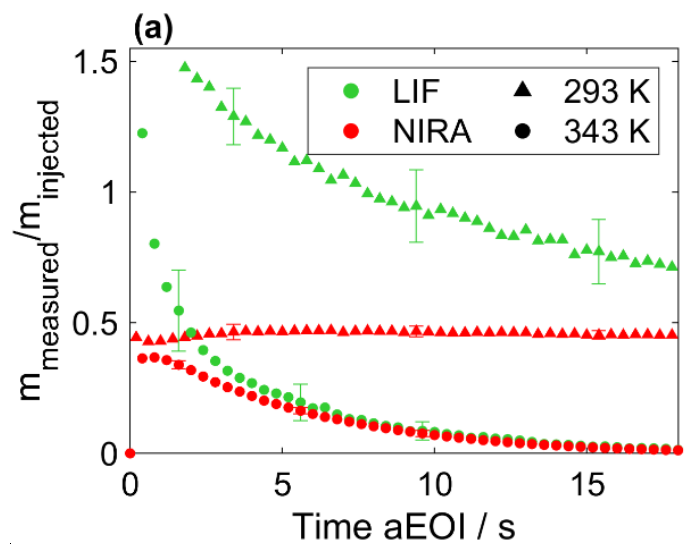

(b)

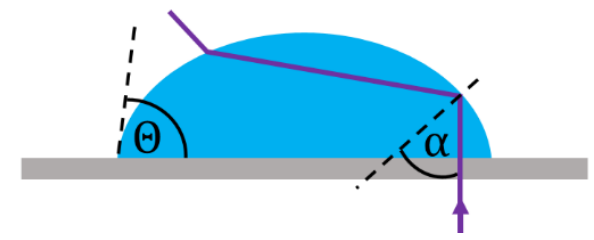

Figure 7. (a) Integrated averaged water masses on hot and cold quartz glass plate, imaged simultaneously by LIF (green) and NIRA (red). Error bars indicate the standard deviation from 20 individual injections. (b) Schematic of total internal reflection of a laser beam inside a droplet.

Especially near the edges of big droplets large contact angles can cause refraction that leads to multiple fluorescence excitation. This is shown in Figure 7 (b), here with the simplified assumption that the laser beam is transmitted vertically through the glass plate. If the contact angle $\theta$ of a droplet is greater than the critical angle $\alpha_{c r}$ for total internal reflection (TIR; $\alpha_{c r}=48.8^{\circ}$ for a water/air interface), at the water/air interface the angle of incidence $\alpha$ of a laser beam may exceed the critical angle. In this case, the laser can be totally reflected inside the droplet, resulting in a longer path of the laser beam in the water. As a result, more fluorescence is excited than accounted for in the calibration. The effect is less likely to occur with small droplets because their contact angle tends to be smaller. This is also indicated by the overestimated LIF thicknesses in Figure 7 (a) at $293 \mathrm{~K}$. Since bigger droplets tend to form at $293 \mathrm{~K}$ than at $343 \mathrm{~K}$ due to higher surface tension and viscosity, TIR occurs in more droplets. Also, Figure 7 (a) shows larger error bars for LIF than for NIRA. This is due to the variation in droplet sizes from injection to injection. 


\section{Conclusions}

Laser-induced fluorescence (LIF) and near-infrared absorption (NIRA) were used to simultaneously image water droplet clusters on a quartz glass plate. For LIF, 0.006 vol.\% anisole was dissolved in water and excited at $266 \mathrm{~nm}$. In preliminary experiments in a closed thin-film cuvette, the LIF signal was found to decrease with a temperature increase, and the signal for a variation in tracer concentration and film thickness was well-described by the BeerLambert law. For NIRA, two cameras were used at wavelengths with high $(1450 \mathrm{~nm})$ and low $(600 \mathrm{~nm})$ absorption by water. This tracer-free method showed good agreement between set and measured film thickness. Only the $1450 \mathrm{~nm}$ detection channel requires a temperature correction in the absorption coefficient. LIF and NIRA water droplet imaging agreed at elevated temperatures and late times after end of injection $(\mathrm{aEOI})$, but otherwise disagree significantly because of several diagnostic artefacts. However, in many applications the relevant surface temperatures are in fact elevated. Especially shortly after aEOI LIF overestimated the thickness compared to NIRA. This could be due to uncertainties in the assumption for the water temperature and light refraction within the droplets.

\section{References}

[1] Schulz, F., Samenfink, W., Schmidt, J., Beyrau, F., 2016, Fuel, 172, pp. 284-292.

[2] Leick, P., Bork, B., Geiler, J. N., July 22.-26. 2018, 14th Triennial International

Conference on Liquid Atomization and Spray Systems.

[3] Greszik, D., 2011, „Entwicklung eines laseroptischen Messverfahrens zur Quantifizierung der Schichtdicke von Wasserfilmen".

[4] Schagen, A., 2015, „Methode der laser-induzierten Lumineszenz zur experimentellen Analyse des Stofftransportes in laminar-welligen Flüssigkeitsfilmen“.

[5] Mirschinka, F., Geiler, J. N., Messina, F., Mansbart, M., Kaiser, S. A., May 17.-19.2021, ILASS-Americas 31st Annual Conference on Liquid Atomization and Spray Systems.

[6] Winter, R., 2011, „Fluorescent Tracers for air-sided Concentration Profile Measurements at the Air-Water Interface".

[7] Volkov, R., Strizhak, P., 2019, Measurement, 135, pp. 231-243.

[8] Schulz, F., Schmidt, J., Beyrau, F., 2015, Experiments in Fluids, 56, 98.

[9] Geiler, J. N., Grzeszik, R, Quaing, S., Manz, A., Kaiser, S. A., 2017, International Journal of Engine Research, 19, pp. 134-147.

[10] Geiler, J. N., 2019, „Bildgebende Messung der Kraftstoffwandfilmdicke durch laserinduzierte Fluoreszenz".

[11] Greszik, D., Yang, H., Dreier, T., Schulz, C., 2011, Applied Optics, 50, A60-A67.

[12] Greszik, D., Yang, H., Dreier, T., Schulz, C., 2011, Applied Physics B, 102, pp. 123-132.

[13] Lubnow, M., Jeffries, J. B., Dreier, T., Schulz, C., 2018, Optics Express, 26, pp. 20902-

20912.

[14] Dupont, J., Mignot, G., Prasser, H., 2015, Experiments in Fluids, 56 (90).

[15] Schulz, C., Sick, V., 2005, Progress in Energy and Combustion Science, 31, pp. 75-121.

[16] Faust, S., Goschütz, M., Kaiser, S. A., Dreier, T., Schulz, C., 2014, Applied

Physics B, 117, pp. 183-194.

[17] Semrock Inc, https://www.semrock.com/FilterDetails.aspx?id=LP02-266RU-25.

[18] Berlman, I. B., 1965, Handbook of Fluorescence Spectra of Aromatic Molecules

[19] Faust, S., Dreier, T., Schulz, C., 2013, Applied Physics B, 112, pp. 203-213.

[20] Tran, K., Guibert, P., Morin, C., Bonnety, J., Pounkin, S., Legros, G., 2015, Combustion and Flame, 162 (10), pp. 3960-3970.

[21] Mueller, T., Wigger, S., Fuesser, H.-J., Kaiser, S. A., 2018, SAE Technical Paper 201801-0634. 